\title{
Erratum to: The Asymptotic Distribution of Self-Normalized Triangular Arrays
}

\author{
David M. Mason
}

Published online: 9 March 2013

(C) Springer Science+Business Media New York 2013

\begin{abstract}
We correct and clarify some ambiguous statements in D. M. Mason (2005): The asymptotic distribution of self-normalized triangular arrays. J. Theoret. Probab., 18, 853-870.
\end{abstract}

\section{Corrections and Clarifications of Mason (2005)}

This note has two purposes. First is to correct some statements in the Introduction and Statements of Results of [4], and second is to provide the result given in Proposition [A] below, which clarifies a claim at the end of the proof of Theorem 2 .

Our corrections are needed since it is not clear that (1.9) always implies (1.2). They are the following:

(i) On page 855 , line 10 , change the "further shows" to "further shows that under the setup of Proposition [A] in this note" and on line 13 change "(1.4)" to "(1.4) nondegenerate".

(ii) On page 855, line 14, replace "or equivalently (1.9) holds" with " $P(V>0)=1$ ".

(iii) On page 856, line 3, replace "Actually" with "Actually under the setup of Proposition $[\mathrm{A}]$ in this note".

We remark in passing that the statements in [4] about triangular arrays of the form $X_{1, n}, \ldots, X_{n, n}, n \geq 1$, are equally valid for triangular arrays of the form $X_{1, n_{k}}, \ldots, X_{n_{k}, n_{k}}, k \geq 1$, where $\left\{n_{k}\right\}_{k \geq 1}$ is an infinite subsequence of the positive integers. Also we point out that everywhere triangular array of infinitesimal independent

The online version of the original article can be found under doi:10.1007/s10959-005-7529-z.

D. M. Mason ( $\varangle)$

Department of Applied Economics and Statistics, University of Delaware, Newark, DE 19716, USA

e-mail:davidm@udel.edu 
random variables should be changed to infinitesimal triangular array of independent random variables, as stated in Proposition [A]. The word infinitesimal was everywhere put in the wrong place.

The following Proposition [A] and Remark 1 justify the claim towards the end of the proof of Theorem 2 on page 868 that says, "This means that every subsequential distributional limit random variable $T$ must be of the form (1.10)." They should have been included in an appendix in the original paper.

Proposition [A] Let $\left\{n_{k}\right\}_{k \geq 1}$ be an infinite subsequence of the positive integers and $X_{1, n_{k}}, \ldots, X_{n_{k}, n_{k}}, k \geq 1$, be an infinitesimal triangular array of independent random variables such that for each $k \geq 1, X_{1, n_{k}}, \ldots, X_{n_{k}, n_{k}}$ are i.i.d. $X_{1, n_{k}}$. Assume that for a necessarily infinitely divisible random variable $U$,

$$
\sum_{i=1}^{n_{k}} X_{i, n_{k}} \rightarrow{ }_{d} U \text {, as } k \rightarrow \infty
$$

Then

$$
\left(\sum_{i=1}^{n_{k}} X_{i, n_{k}}, \sum_{i=1}^{n_{k}} X_{i, n_{k}}^{2}\right) \rightarrow_{d}(U, V) \text {, as } k \rightarrow \infty
$$

where the two dimensional infinitely divisible random vector $(U, V)$ in (1.2) has the representation:

$$
(U, V)={ }_{d}\left(b+W+\tau Z, S+\tau^{2}\right)
$$

with $b$ and $\tau \geq 0$ being suitable constants,

$$
\begin{aligned}
W= & \int_{0}^{1} \varphi_{1}(s) d N_{1}(s)+\int_{1}^{\infty} \varphi_{1}(s) d\left\{N_{1}(s)-s\right\} \\
& -\int_{0}^{1} \varphi_{2}(s) d N_{2}(s)-\int_{1}^{\infty} \varphi_{2}(s) d\left\{N_{2}(s)-s\right\}
\end{aligned}
$$

and

$$
S=\int_{0}^{\infty} \varphi_{1}^{2}(s) d N_{1}(s)+\int_{0}^{\infty} \varphi_{2}^{2}(s) d N_{2}(s),
$$

with $N_{1}$ and $N_{2}$ being independent right continuous Poisson processes on $[0, \infty)$ with rate $1, Z$ being a standard normal random variable independent of $N_{1}$ and $N_{2}$, and $\varphi_{1}$ and $\varphi_{2}$ being two left continuous, nonincreasing, nonnegative functions defined on $(0, \infty)$ satisfying for all $\delta>0$,

$$
\int_{\delta}^{\infty} \varphi_{i}^{2}(s) d s<\infty \text { for } i=1,2
$$


Proof The proof that (1.1) implies (1.2) follows along very similar lines to that of Lemma 4 in [2]. To relieve the notational burden, in the following we shall write $n=n_{k}$. By parts (ii) and (iii) of Theorem 4.7 on page 61 of [1], the distributional convergence (1.1) implies that there exists a Lévy measure $\mu$ such that for every $\delta>0$ such that $\mu\{-\delta, \delta\}=0$,

$$
\begin{aligned}
w & -\lim _{n \rightarrow \infty} \sum_{i=1}^{n} n \mathcal{L}\left(X_{i, n}\right) \mid(|x|>\delta) \\
& =w-\lim _{n \rightarrow \infty} n \mathcal{L}\left(X_{1, n}\right)|(|x|>\delta)=\mu|(|x|>\delta)
\end{aligned}
$$

and for some $a_{\delta}$

$$
\lim _{n \rightarrow \infty} E S_{n, \delta}=\lim _{n \rightarrow \infty}\left(n E X_{1, n, \delta}\right)=a_{\delta}
$$

where

$$
S_{n, \delta}:=\sum_{i=1}^{n} X_{i, n} 1\left\{\left|X_{i, n}\right| \leq \delta\right\}=: \sum_{i=1}^{n} X_{i, n, \delta} .
$$

(Note that we use here the notation of [1].) Now by part (i) of the same theorem, (1.1) also implies that for some $0 \leq \sigma^{2}<\infty$,

$$
\lim _{\delta \searrow 0}\left\{\begin{array}{l}
\limsup _{n \rightarrow \infty} \\
\liminf _{n \rightarrow \infty}
\end{array}\right\} \sum_{i=1}^{n} E\left(X_{i, n, \delta}-E X_{i, n, \delta}\right)^{2}=\sigma^{2} .
$$

Notice that

$$
\sum_{i=1}^{n} E\left(X_{i, n, \delta}-E X_{i, n, \delta}\right)^{2}=n E X_{1, n, \delta}^{2}-n^{-1}\left(n E X_{1, n, \delta}\right)^{2}
$$

Further by (1.8) for every $\delta>0$ such that $\mu\{-\delta, \delta\}=0, n^{-1}\left(n E X_{1, n, \delta}\right)^{2} \rightarrow 0$, which by (1.10) and (1.11), implies

$$
\lim _{\delta \searrow 0}\left\{\begin{array}{l}
\lim _{\sup _{n \rightarrow \infty}} \\
\liminf _{n \rightarrow \infty}
\end{array}\right\} E V_{n, \delta}=\sigma^{2}
$$

where

$$
V_{n, \delta}:=\sum_{i=1}^{n} X_{i, n}^{2} 1\left\{\left|X_{i, n}\right| \leq \delta\right\}=\sum_{i=1}^{n} X_{i, n, \delta}^{2}
$$


with

$$
E V_{n, \delta}=n E X_{1, n, \delta}^{2}=\int_{|x| \leq \delta} n x^{2} d \mathcal{L}\left(X_{1, n}\right)
$$

Now let $\delta_{m}, m \geq 1$, be a sequence of constants converging to zero such that $0<$ $\delta_{m+1}<\delta_{m}<\delta_{0}=\delta$, and $\mu\left\{-\delta_{m}, \delta_{m}\right\}=0, m \geq 0$. Then for each $m \geq 1$, by (1.7) and $\mu\left\{-\delta_{m}, \delta_{m}\right\}=\mu\{-\delta, \delta\}=0$,

$$
\begin{aligned}
& \liminf _{n \rightarrow \infty} \int_{|x| \leq \delta_{m}} n x^{2} d \mathcal{L}\left(X_{1, n}\right)+\int_{\delta_{m}<|x| \leq \delta} x^{2} d \mu(x) \\
& =\liminf _{n \rightarrow \infty} \int_{|x| \leq \delta_{m}} n x^{2} d \mathcal{L}\left(X_{1, n}\right)+\lim _{n \rightarrow \infty} \int_{\delta_{m}<|x| \leq \delta} n x^{2} d \mathcal{L}\left(X_{1, n}\right) \\
& =\liminf _{n \rightarrow \infty} \int_{|x| \leq \delta} n x^{2} d \mathcal{L}\left(X_{1, n}\right) \leq \limsup _{n \rightarrow \infty} \int_{|x| \leq \delta} n x^{2} d \mathcal{L}\left(X_{1, n}\right) \\
& =\limsup _{n \rightarrow \infty} \int_{|x| \leq \delta_{m}} n x^{2} d \mathcal{L}\left(X_{1, n}\right)+\lim _{n \rightarrow \infty} \int_{\delta_{m}<|x| \leq \delta} n x^{2} d \mathcal{L}\left(X_{1, n}\right) \\
& =\limsup _{n \rightarrow \infty} \int_{|x| \leq \delta_{m}} n x^{2} d \mathcal{L}\left(X_{1, n}\right)+\int_{\delta_{m}<|x| \leq \delta} x^{2} d \mu(x) .
\end{aligned}
$$

Now by letting $m \rightarrow \infty$, we see by (1.12) that

$$
\lim _{n \rightarrow \infty} \int_{|x| \leq \delta} n x^{2} d \mathcal{L}\left(X_{1, n}\right)=\sigma^{2}+\int_{0<|x| \leq \delta} x^{2} d \mu(x)=: b_{\delta} .
$$

Moreover, we get from (1.15) that for every $k>2$,

$$
\lim _{\delta \searrow 0} \limsup _{n \rightarrow \infty} \int_{|x| \leq \delta} n|x|^{k} d \mathcal{L}\left(X_{1, n}\right)=0
$$

We now proceed as in the proof of Lemma 4 in [2]. We see using (1.16) that for any $\alpha, \beta \in \mathbb{R}$,

$$
\lim _{\delta \searrow 0}\left\{\begin{array}{l}
\lim _{\sup _{n \rightarrow \infty}} \\
\liminf _{n \rightarrow \infty}
\end{array}\right\} E\left(\alpha\left(S_{n, \delta}-E S_{n, \delta}\right)+\beta\left(V_{n, \delta}-E V_{n, \delta}\right)\right)^{2}=\alpha^{2} \sigma^{2} .
$$

Write $\rho=\mu \circ T^{-1}$, where $T(x)=\left(x, x^{2}\right)$. Clearly by (1.7) for every $\delta>0$ such that $\mu\{-\delta, \delta\}=0$

$$
w-\lim _{n \rightarrow \infty} n \mathcal{L}\left(X_{1, n}, X_{1, n}^{2}\right)|(\|x\|>\delta)=\rho|(\|x\|>\delta) .
$$

Furthermore, by (1.8) and (1.15) we have for every $\delta>0$ such that $\mu\{-\delta, \delta\}=0$

$$
\left(E S_{n, \delta}, E V_{n, \delta}\right) \rightarrow\left(a_{\delta}, b_{\delta}\right)
$$


Thus by the central limit theorem in $\mathbb{R}^{2}$ on pp. 67-68 of [1] and arguing just as in [2] we get that (1.2) holds with $(U, V)$ having characteristic function $E \exp (s U+t V)=$

$$
\exp \left\{-\frac{\sigma^{2} s^{2}}{2}+i\left(a_{\delta} s+\sigma^{2} t\right)+\int\left(\exp \left(i\left(s u+t u^{2}\right)\right)-1-i s u 1\{|u| \leq \delta\}\right) d \mu(u)\right\},
$$

for any $\delta>0$ such that $\mu\{-\delta, \delta\}=0$. It can be shown using Proposition 5.7 in [3] that a pair of random variables $(U, V)$ with this characteristic function has the distributional representation (1.3) where $\tau^{2}=\sigma^{2}$ and $b$ is a suitable constant. It is shown there how $\varphi_{1}$ and $\varphi_{2}$ are defined via the Lévy measure $\mu$.

Remark 1 We note that if $X$ is in the centered Feller class with $a_{n}$ an appropriate sequence of norming constants and $X_{1}, X_{2}, \ldots$, are i.i.d. $X$, then for every subsequence of $\{n\}$ there exists a further subsequence $\left\{n_{k}\right\}$ such that the triangular array $X_{i, n_{k}}=X_{i} / a_{n_{k}}, 1 \leq i \leq n_{k}, k \geq 1$, satisfies (1.1), with $U$ nondegenerate, and thus (1.2) and (1.19) hold, as was pointed out in [2]. Also we mention that it can be inferred using the Theorem in [5] that necessarily " $P(V>0)=1$ ".

Remark 2 A special case of the Proposition 1 implies that for any triangular array $X_{1, n_{k}}, \ldots, X_{n_{k}, n_{k}}, k \geq 1$, satisfying its assumptions, and

$$
\sum_{i=1}^{n_{k}} X_{i, n_{k}} \rightarrow{ }_{d} N\left(0, \sigma^{2}\right), \quad \text { as } k \rightarrow \infty
$$

then

$$
\sum_{i=1}^{n_{k}} X_{i, n_{k}}^{2} \rightarrow_{P} \sigma^{2}, \quad \text { as } k \rightarrow \infty
$$

Acknowledgments The author thanks Evarist Giné for checking the proof of Proposition [A], as well as the referee for a careful reading of the manuscript.

\section{References}

1. Araujo, A., Giné, E.: The Central Limit Theorem for Real and Banach Valued Random Variables. Wiley Series in Probability and Mathematical Statistics. Wiley, New York (1980)

2. Giné, E., Mason, D.M.: On the LIL for self-normalized sums of IID random variables. J. Theoret. Probab. 11, 351-370 (1998)

3. Maller, R., Mason, D.M.: Small-time compactness and convergence behavior of deterministically and self-normalised Lévy processes. Trans. Am. Math. Soc. 362, 2205-2248 (2010)

4. Mason, D.M.: The asymptotic distribution of self-normalized triangular arrays. J. Theoret. Probab. 18, 853-870 (2005)

5. Pruitt, W.E.: The class of limit laws for stochastically compact normed sums. Ann. Probab. 11, 962-969 (1983) 